\title{
Qualitative Physics in Virtual Environments
}

\author{
Marc Cavazza, Simon Hartley, Jean-Luc Lugrin, Mikael Le Bras \\ School of Computing, University of Teesside \\ Middlesbrough TS1 3BA United Kingdom \\ \{m.o.cavazza, s.hartley, j-1.lugrin, m.lebras\}@tees.ac.uk
}

\begin{abstract}
In this paper, we describe a new approach to the creation of virtual environments, which uses qualitative physics to implement object behaviour. We adopted Qualitative Process Theory as a qualitative reasoning formalism, due to its representational properties (e.g., its orientation towards process ontologies and its explicit formulation of process' pre-conditions). The system we describe is developed using a game engine and takes advantage of its event-based system to integrate qualitative process simulation in an interactive fashion. We use a virtual kitchen as a test environment. In this virtual world, we have implemented various behavioural aspects: physical object behaviour, complex device behaviour (appliances) and "alternative" (i.e. non-realistic) behaviours, which can all be simulated in user real-time. After a presentation of the system architecture and its implementation, we discuss example results from the prototype. This approach has potential applications in simulation and training, as well as in entertainment and digital arts. This work also constitutes a test case for the integration of an Artificial Intelligence technique into 3D user interfaces.
\end{abstract}

Categories and Subject Descriptors

H5.1 [Multimedia Information Systems] Artificial, Augmented and Virtual Reality

\section{General Terms}

Theory, Algorithms, Design and Experimentation

Keywords:

Modelling and Simulation, Intelligent Virtual Environments, Qualitative Physics.

\section{INTRODUCTION AND RATIONALE}

The description of objects' behaviours in Virtual Environments (VEs) is faced with a number of challenges. On one hand, real-time physical simulation requires significant computing resources.
On the other hand, VEs and the applications they support emphasises user-centred aspects such as interaction, agency and causality and the potential to generate explanations; behavioural mechanisms should thus support these aspects as well. In this paper, we present primary results from the integration of Qualitative into VEs Physics [13]. There are several reasons that make Qualitative Physics a promising approach to describe the behaviour of objects in virtual worlds. Firstly, it can be used to simplify the description of behaviours in simulation, where analytical computations might prove complex. Secondly, it is particularly good at aggregating the behaviour of complex devices, such as engines, appliances and mechanical devices that can be part of various simulation or training virtual environments. Thirdly, it supports the principled creation of behaviours through the definition of process ontologies [7]. Finally, it offers new possibilities of integration and explanation that could be accessible through user interaction, while preserving a visually realistic setting.

The logic of most virtual environments is to operate on a discretised event model: our original insight was that this should provide a path for the integration of the discretised simulations of Qualitative Physics. Many recent interactive systems are based on the notion of event for their implementation: Virtual Reality systems [9] and game engines in particular, intensively exploit this notion for their implementation. The event-based model is adapted to the user-centred natured of the environment, as it supports the propagation of the consequences of user interventions. On the other hand, the notion of event is also the basis for the high-level description of physical behaviour, as events discretise the continuous motion of objects (in terms of positions, trajectories, contacts with other objects) into meaningful high-level actions (such as colliding, touching, entering volumes or areas).

In this paper, we describe an approach integrating qualitative physics into a 3D interactive environment, which should support a variety of applications in simulation and training. After a brief overview of the system, we discuss the integration of qualitative process theory with the event-based system of the environment. We give several examples illustrating different categories of behaviour, in traditional physics and device behaviour. Finally, we discuss potential applications of Qualitative Physics in Virtual Environments. 


\section{SYSTEM OVERVIEW}

The system presents itself as a $3 \mathrm{D}$ environment derived from the Unreal Tournament 2003 game engine. The rationale for using a game engine in research is that it supports advanced graphic rendering and animation control as well as the integration of external software modules, which makes it an ideal development environment [10]. This environment supports several interaction mechanisms in terms of object manipulation (which can be grabbed, moved, so as to create physical situations) as well as triggers that can start certain processes associated with the environment's devices (heating, cooling, flowing, etc.).

Most physical objects in the environment can be involved in a variety of pre-defined Qualitative Processes (QPs). These will be triggered upon interaction with the objects, which satisfies the pre-conditions of some relevant QP (for instance, when a container is aligned with a liquid flow). The environment remains interactive while QPs are active, as QPs themselves are simulated in user real-time, updating the environment's objects' properties as landmark values are reached for physical properties. Depending on their type, QPs define an "implicit time" corresponding to the set of landmark values and the mechanisms by which they are reached: for instance, heat transfers take place on a longer time scale than other physical events, e.g. involving object motion.

The effects of the simulation are perceived in the environment through two main mechanisms: i) the alteration of the objects themselves, which has a visual translation in terms of their appearance. For instance, recipients will be filled by flows of liquids, objects would melt, other liquids would evaporate or freeze, etc. ii) a change in the physical properties of objects, which will only become apparent during the course of further physical interactions with these objects. For instance, the filled container will become heavier, or an object would be become brittle as a result of being heated dry.

As a demonstration environment, we have created a virtual kitchen, which is an ideal environment to represent many physical processes, from filling recipients to heat transfer (heating, boiling, cooling, refrigerating), also including some elementary mechanics (rolling, sliding/slipping, falling). The layout of the virtual kitchen is presented on Figure 1.

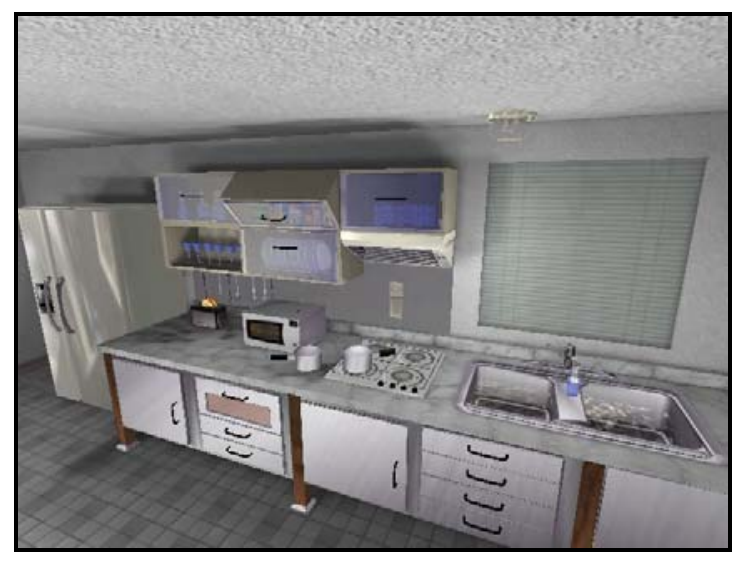

Figure 1:The Virtual Kitchen Environment

\section{USING QUALITATIVE PROCESS THEORY IN VIRTUAL ENVIRONMENTS}

Of the various approaches that have been described in qualitative physics, we have opted for Qualitative Process Theory (henceforth QPT) [6], essentially for its representational properties. QPT descriptions are centred on physical processes (e.g. liquid flows, heat transfer, etc.) whose states are described through the values of qualitative variables. Relations between variables are described through influence equations and qualitative proportionalities. The former correspond to the actual dynamics of the process, the causal evolution; for instance, that the amount of liquid in a recipient increases with the inflow. The latter maintain "static" relationships between variables, such as the fact that the mass of liquid in the container is proportional to its volume.

The QPT formalism is well adapted to its integration in virtual environments, for several reasons: i) the explicit description of a QP's pre-conditions supports the definition of procedures activating the QP simulation from physical events involving objects in the virtual world. This is the basic mechanism for integration of QPs in the interactive environment, ii) the relations governing variables' evolution (influence equations and qualitative proportionalities) are encapsulated within the $\mathrm{QP}$ description. This makes it possible to simulate the process locally, without having to solve a set of confluence equations or search a network of constraints relating variables associated with different objects in the environment, iii) The kind of causality associated with QP descriptions [7] can be matched to user interventions, and iv) QPT has been successfully used to define ontologies with a significant number of processes: in our context this could facilitate "worldmaking". 


\subsection{Example QPS}

We have defined various categories of QPs corresponding to physical processes involving individual objects, or to those describing the behaviour of certain devices, which often involve several integrated processes. QPs have been formalised in QPT representations as illustrated in Figure 2 below.

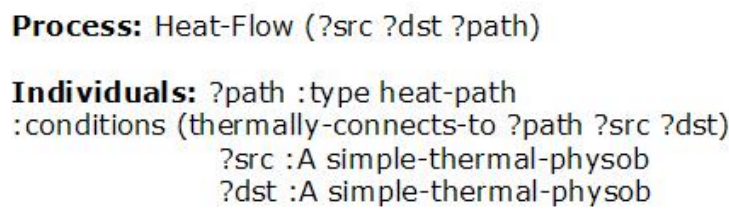

Individuals: ?path :type heat-path

:conditions (thermally-connects-to ?path ?src ?dst) ?src : A simple-thermal-physob ?dst :A simple-thermal-physob

Preconditions: heat-aligned ?path

Quantity Conditions: (A(temperature ?src) > (A(temperature ?dst ))

Relations:

(Quantity heat-flow-rate) $\alpha$ (A(temp ?src)- (A(temp ?dst ))

Influences: I+ (heat ?dst) (A heat-flow-rate) I- (heat ?src) (Aheat-flow-rate)

\section{Figure 2: A "Heat-Transfer" Qualitative Process}

In terms of their actual implementation, pre-conditions are encoded in specific UnrealScript ${ }^{\mathrm{TM}}$ (the programming language of UT2003 serving as an API) procedures associated to the virtual world objects' in order to trigger the activation of relevant QPS. In that sense, pre-conditions are not strictly speaking part of the actual QP representation implemented. However, all the other elements of the QP representations; qualitative variables, qualitative proportionalities and influence equations are implemented within the QP engine. Their actual use by the engine during simulations is discussed in the following sections.

\section{SYSTEM ARCHITECTURE AND IMPLEMENTATION}

The system comprises a graphic environment, composed of the UT 2003 engine and an external qualitative simulation module (QP engine), developed in $\mathrm{C}++$. The software architecture is based on TCP/IP communication, supported through the TCPLink class in UT 2003. The messages exchanged between the UT 2003 environment and the QP engine correspond, on one side, to the activation conditions of various QP instances run by the engine. On the other side, the QP engine sends messages to update object states, which are interpreted by the Unreal Environment (Figure3).

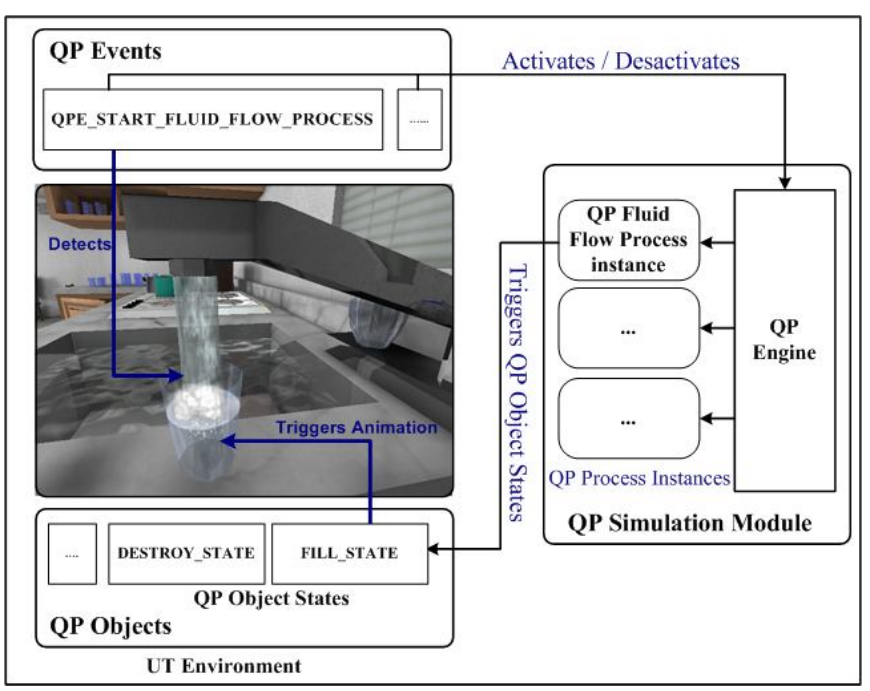

Figure 3: System Architecture

\subsection{The Event System}

As we have introduced earlier, the concept underlying the integration of qualitative physics in virtual environment is the discretisation of the environment behaviour through the definition of system events. The Unreal Tournament engine extensively relies on event generation to support many of its interaction aspects and, most importantly, the mechanism for event generation is accessible to redefine specific behaviours. Formally, an event can be characterised as an encapsulated message, which is generated by an Event Source, this being an object of the environment. Examples of such basic events are: Bump (Actor Other), Touch (Actor Other), UnTouch (Actor Other), ActorEnteredVolume (Actor (ther), etc.

The Unreal Tournament Engine implements two different kinds of event: the basic (primitive) events, which are lowlevel events defined within the game engine (derived from the collision detection procedures in the graphic engine), and the programmed events. The latter are events whose definitions are scripted and can thus be programmed by the system developer. They can be expressed as the conjunction of basic events and various tests on the objects involved in these events, for instance the contact between two objects, one being a recipient and the other one a fluid flow (see Figure 6). We have called these "QP events"(QPE).

From another perspective, basic events can be classified as discrete or continuous events. Discrete events notify instantaneous actions, such as "bumping", while continuous events signal the beginning and ending of durative actions, for instance touch/untouch, attach/unattach (signaling that physical objects are being transported or manipulated). Within QP events, these can detect QP activation as well as QP interruption/termination, when its pre-conditions cease to be valid. The generation of QP events for the activation of QPs is presented in Figure 4. : From the QP's pre- 
conditions, QP events are attached to objects, testing the validity of these pre-conditions and triggering the activation or the termination of the QP simulation.

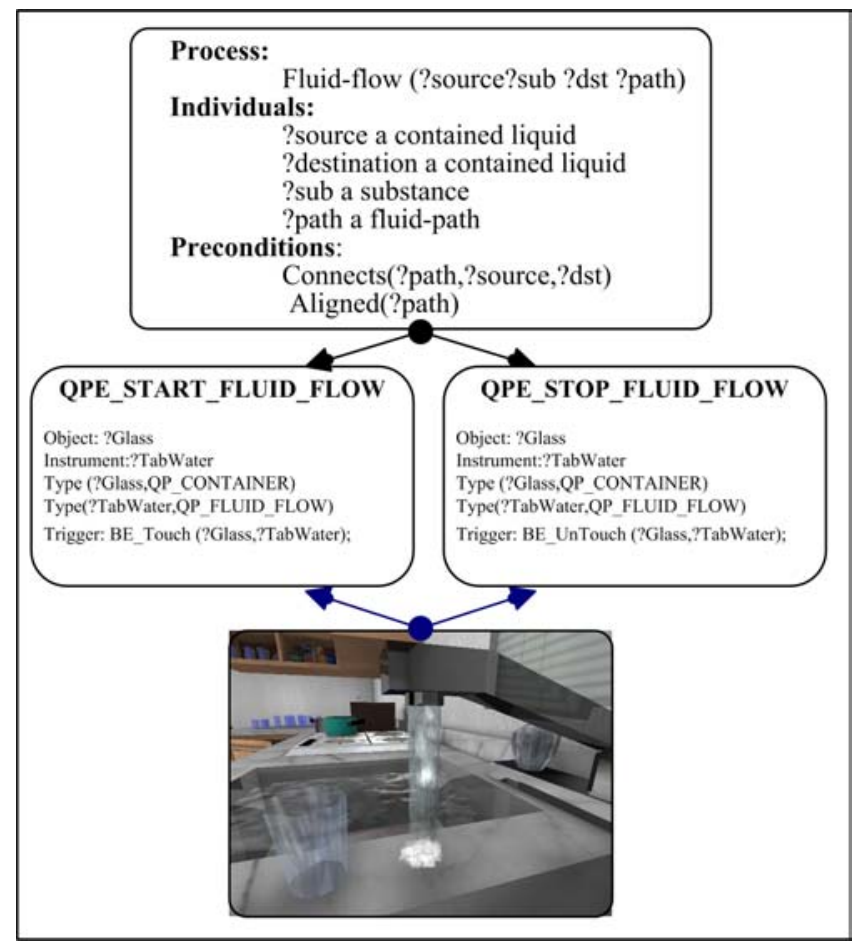

Figure 4: Generation of QP Events

\subsection{The notion of QP objects}

The integration of Qualitative Physics in the virtual environments' basic mechanisms is achieved through the redefinition of a special class of physical objects: qualitative process objects, or QP objects. This follows traditional implementation techniques by which classes of objects are defined depending on the computations they can trigger from the interactions they participate in (i.e., in UT 2003 , objects manipulated by the native physics engine, $\mathrm{Karma}^{\mathrm{TM}}$, are defined as members of the class of "Karma"TM objects").

The QP objects have several properties: i) they are associated with an event interception mechanism that attaches data-driven procedures for the recognition of the pre-conditions of QPs in which they can take part (Figure 4 ), ii) their properties can be defined through qualitative variables, which are the key variables defined within QPs and are involved in qualitative proportionalities and influence equations, iii) they are associated states that have a visual translation, including in terms of transitions between states (e.g. animations showing a recipient filling, a liquid evaporating, etc.). These states correspond to landmark values for the qualitative variables

\subsection{The QP engine}

Qualitative Simulation is controlled by the "QP Engine". At initialisation time, every object that can take part in QPs is associated QP events for their activation. Upon satisfaction of QP's preconditions, these events will activate an instance of that $\mathrm{QP}$ in the $\mathrm{QP}$ engine. On activation, the quantity conditions for the process are tested on the values that the objects of the world have, which have been passed to the qualitative engine.

The main mechanism of the QP engine consists in the resolution of influence equations. The algorithm for the solution of influences within the Qualitative Process system proceeds by:

1. Testing if the Quantity to be changed is being directly or indirectly influenced.

2. If the Quantity is being influenced, its derivative value is calculated by the summation of all the influences. The first stage is to test whether all the influences have the same sign. If all the influences have the same sign the system can determine readily how the quantity will change.

3. Performing the resolution of ambiguous influences by testing if any process effects are significantly greater than others. However, in our current implementation, this has only been used in a limited number of situations, so we cannot claim that our solution is exhaustive. The method of ratios is also implemented: in this method the influences on the numerator and denominator are paired up and the net influence of each pair is resolved separately.

The system updates values as the qualitative simulation passes the landmarks that have been given to the objects (it uses qualitative proportionalities where appropriate). The landmarks are added to the objects and when the QP Engine changes a value within an object ii also tests whether the change triggers the event for passing the landmark. The qualitative engine then generates appropriate events in the unreal engine that trigger corresponding changes in objects' states.

\subsection{Support for Interactivity}

The integration of Qualitative Physics in virtual environment should preserve their interactive nature. This means that QPs can run as background processes (for instance representing devices embedded in the environment, such as refrigerators, central heating, etc.) or can be triggered by physical interventions initiated by the user. Think for instance of turning on a gas hob, putting a kettle on it, etc. User interaction should also at any time be able to interrupt QPs; for example, removing a glass from under the running tap would stop the filling process at its current level. This is achieved through the QP events associated with world's objects. It uses a property of durative basic events to be described in pairs, e.g., touch/untouch. If the recognition of a QP's precondition is based on a touch event involving certain object classes (such as a heat source and an object), the detection of an untouch event involving the same object instances that have triggered the QP should thus interrupt it. 


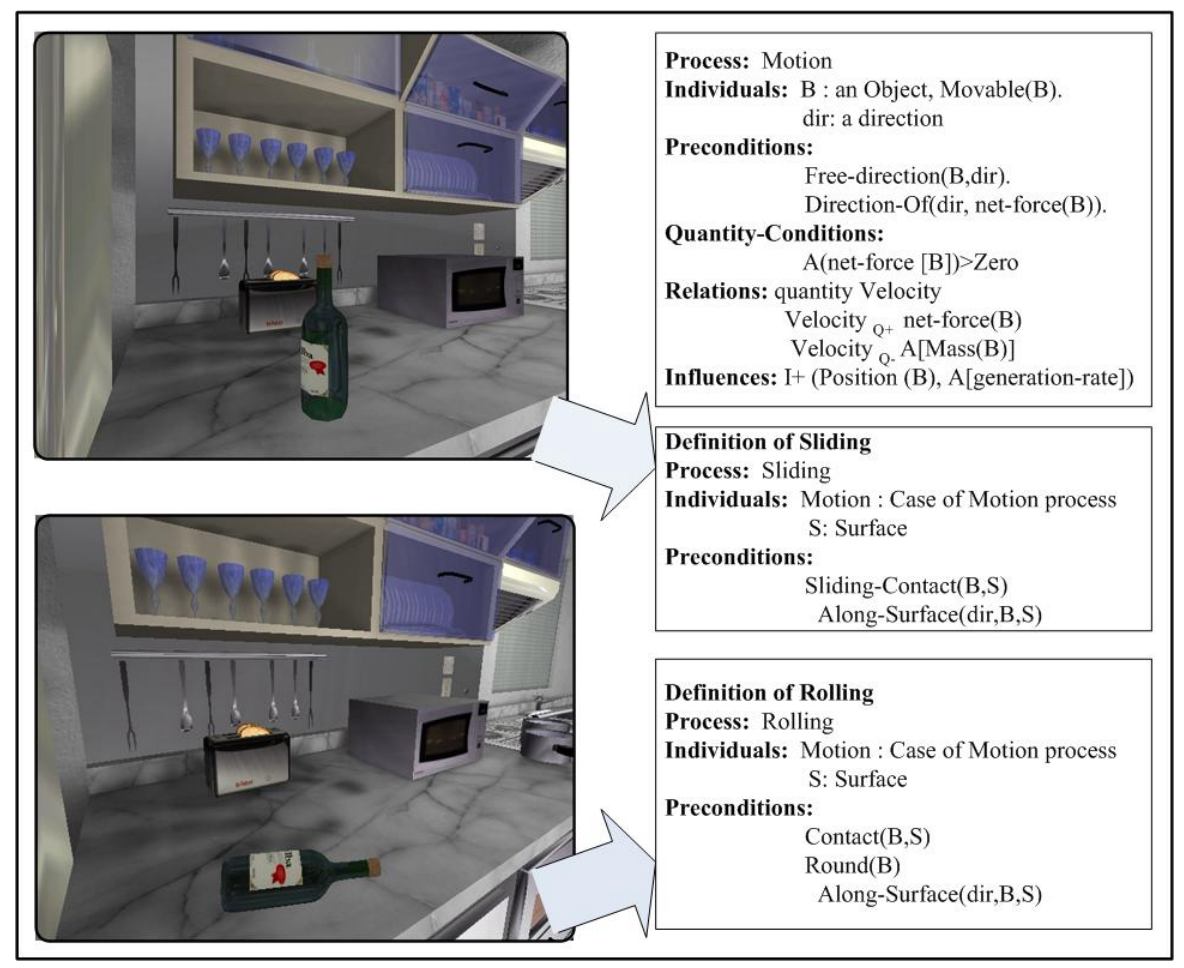

Figure 5:Elementary Physical Behaviour: Rolling or Sliding

\section{EXAMPLE RESULTS}

This use of qualitative physics has clear advantages in the context of VEs, which is to avoid complex numerical computations, where an object behaviour can be easily grasped in a qualitative fashion. A first example, where QPT is an alternative to solving mechanics equations, is to determine whether a bottle or any cylindrical object would be rolling or sliding, which could otherwise involve fairly complex numerical computations [5][6]. To a large extent, similar approaches are part of game physics programming: however, QPT provides a principled way for such descriptions, which also supports a consistent integration of various types of processes. Figure 5 shows how the motion behaviour (rolling versus sliding) is determined by different pre-conditions (actually, an additional condition for rolling, which can be tested by the QP event).

Another example, well adapted to the simulation of physical phenomena in a virtual world, deals with liquid flows [6]. Figure 6 shows the behaviour of the system simulating the filling of a glass from a running tap. When objects, which can behave as recipients, are aligned with a liquid flow (here the kitchen tap), this, corresponding to the pre-condition of a filling process, activates the corresponding liquid-flow QP on these objects. The process running in the QP engine updates the value of the amount of water in the glass, through its influence equations. In a similar fashion, qualitative proportionalities update the total mass of the glass, as well as the height of liquid. These variables transform the state of the filling glass in the virtual world by updating its physical properties (e.g. weight) as well as its appearance. The overall dynamics is dictated by the QP simulation process, the speed of the filling glass animation being an approximation of these dynamics. As described in the previous sections, at any time, the user can remove the glass from the running tap, which will interrupt the process while retaining the physical properties of the glass (amount of water filled into the glass) 


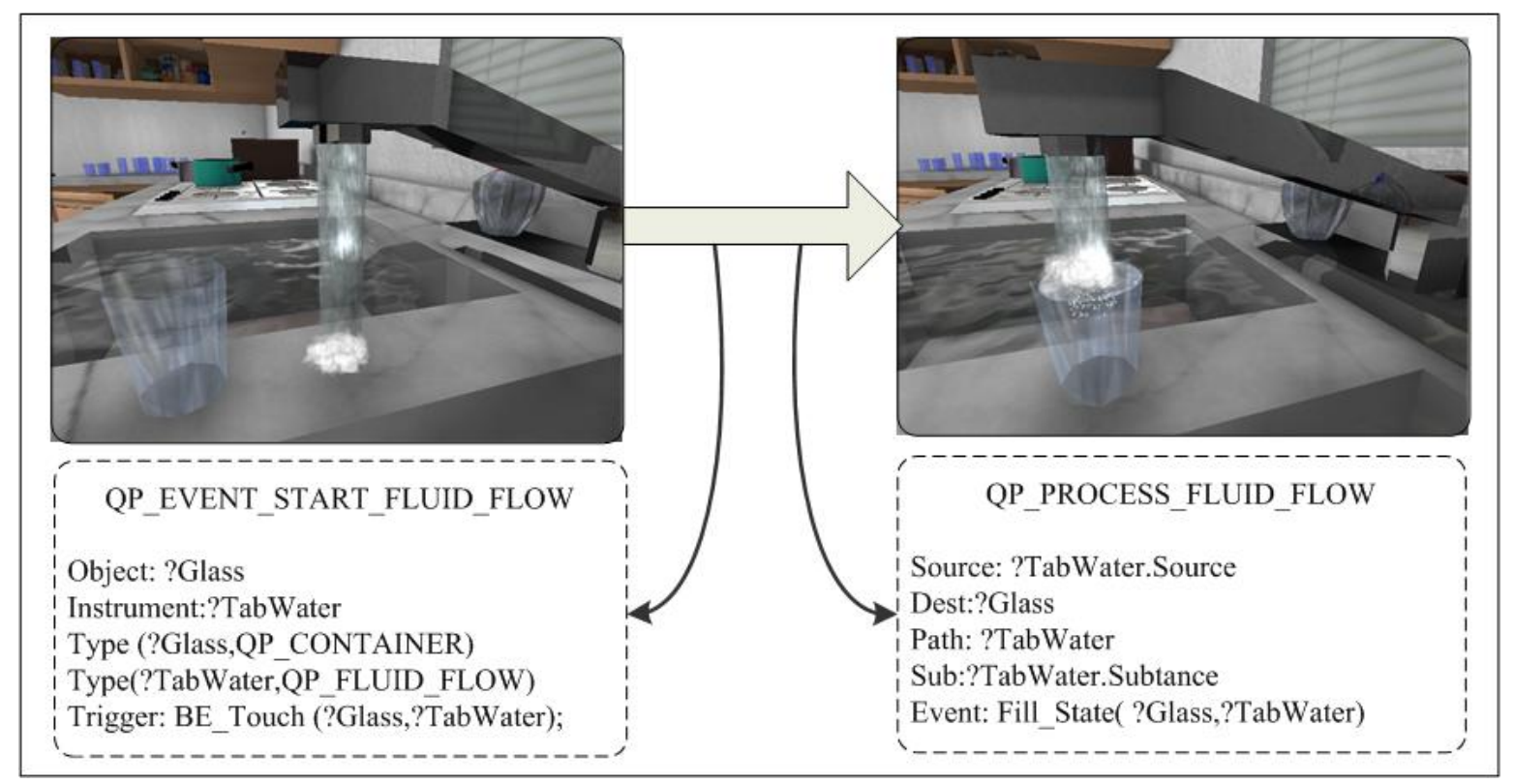

Figure 6:Liquid Flows: Filling a Glass with Running Water

One of the specific advantages of qualitative physics over numerical simulations is its capacity to describe the behaviour of complex devices. We have illustrated this by modelling the behaviour of a refrigerator, which involves a total of six qualitative processes [4][12]: fluid flow, evaporation, heat-flow-inside (evaporator), pumped-gas-flow, heat-flow-outside (condenser) and condensation.

The first two processes, evaporation and fluid flow, perform at the same rate giving a constant volume of liquid within the system thus in order to maintain a constant temperature a heat flow from inside the refrigerator must exist to balance the difference between the heat from the liquid which is entering the evaporator and the heat which is carried away by the evaporated gas. This then means that there is a heat flow process between the inside of the fridge and the evaporator. The heat-flow-inside (evaporator), which is the heat-flow process between the inside of the fridge and the evaporator can be connected to any object entering the fridge.

For instance if we take a dish out of the oven and put it to cool down in the refrigerator (see Figure 7), the object becomes involved in the internal heat-transfer process. In implementation terms, the heat transfer between the object and the refrigerator is triggered by the object entering the refrigerators volume (basic events such as ActorEnteredVolume (Actor Other) are used for detecting that kind of event).

\section{APPLICATIONS AND PERSPECTIVES}

The integration of qualitative physics to virtual environments has a significant potential for applications in several different contexts:
- Some Virtual Environments for the teaching of Physics. QPT represents physical processes and physical laws, it can be used to teach and illustrate the principles of physics through the creation of virtual labs or virtual physics classes. The advantage here lies in the $3 \mathrm{D}$ interactivity with objects that makes possible to experience physics "hands-on", for instance by setting up experiments in elementary thermodynamics or the mechanics of solid objects.

- Simulation and Training. The fact that a virtual environment could include a qualitative model of a device, while allowing interaction with its constituents can be used for various training tasks, such as maintenance [11]. We have previously developed a similar approach in a medical application, which used qualitative physiological models to simulate the condition of a virtual patient [2]. However, this kind of application probably needs to be extended to include explanatory capabilities.

- Virtual Reality Art. This unusual application is actually a central component of the project within which this research takes place, as it constitutes its original target application [3]. Its rationale is that the creation of virtual worlds in VR Art should be extended to include non-realistic behaviour violating the traditional laws of Physics to create fantasy worlds (that still require consistency in their properties). One way to redefine laws of Physics in a principled fashion being to define "alternative" qualitative processes. 


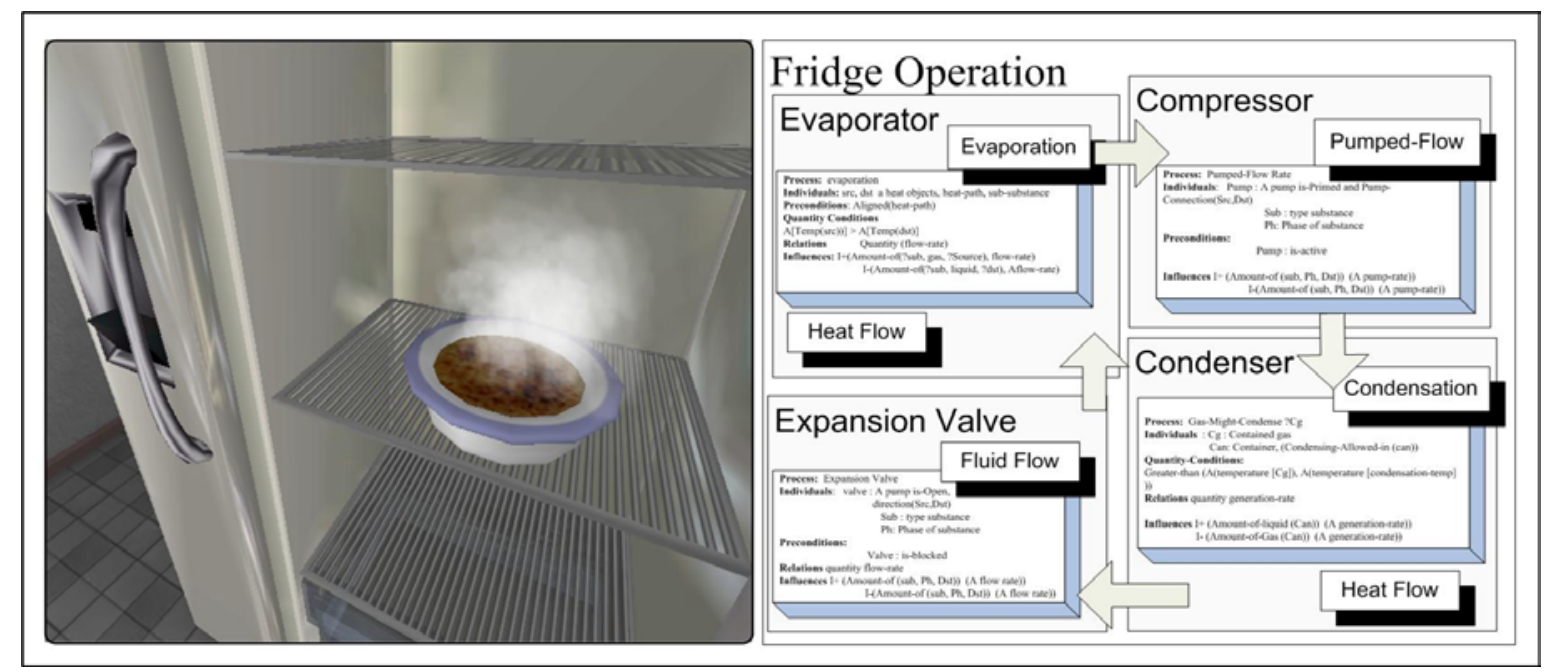

Figure 7:Heat Transfer in the Refrigerator

\section{CONCLUSIONS}

We have presented a first integration of Qualitative Physics in Virtual Environments, based on Qualitative Process Theory. From an implementation perspective, the discretisation of Physics in game engines, through their event-based systems, provides a sound basis on which to integrate the discrete simulations of qualitative physics.

A well-recognised advantage of qualitative physics is that its CPU requirements are much smaller than those of numerical simulations. However, this is also the case because, for the kind of QPs we have formalised, we did not require complex envisionment procedures.

Yet, the most important conclusion lies in the diversity of behaviours that are amenable to qualitative descriptions in virtual environments. In a similar fashion, an additional conclusion of this research is that the representational properties of QPT have proven well adapted to the context of interactive systems.

This work also constitutes a test case for the integration of an Artificial Intelligence technique into 3D user interfaces [1]: in future developments, Qualitative Physics should be able to support the principled integration, over the whole virtual environment, of a large number of diverse behavioural descriptions.

\section{ACKNOWLEDGEMENTS}

This work has been funded in part by the European Commission through the ALTERNE project (IST-38575). Original level design for the kitchen environment by VSK Klan.

\section{REFERENCES}

1. Aylett, R. and Cavazza, M., 2001. Intelligent Virtual Environments: a State-of-the-Art Report. In: Eurographics 2001, STAR Reports volume.

2. Cavazza, M. and Simo, A., 2003. A Virtual Patient Based on Qualitative Simulation. $A C M$ Intelligent User Interfaces 2003, Miami, FL, USA, pp. 19-25

3. Cavazza, M., Hartley, S., Lugrin, J.-L. and Le Bras, M., 2002. Alternative Reality: Qualitative Physics for Digital Arts, Qualitative Reasoning Workshop 2003, Brasilia, Brasil.

4. Collins, J. and K. Forbus. (1989). Building qualitative models of thermodynamic processes. Proceedings of the Qualitative Reasoning Workshop.

5. De Kleer, J. Qualitative and Quantitative knowledge in classical mechanics. TR-352, MIT AI Lab, Cambridge, 1975.

6. Forbus, K.D., Qualitative Process Theory, Artificial Intelligence, 24, 1-3, pp. 85-168, 1984.

7. Forbus, K.D., Qualitative Reasoning. In A.B. Tucker, editor, The Computer Science and Engineering Handbook, pp. 715-733. CRC Press, 1996.

8. Jacobson, J. and Hwang, Z. 2002 Unreal Tournament for Immersive Interactive Theater. Communications of the ACM, Vol. 45, 1, pp. 3942.

9. Jiang, H., Kessler, G.D and Nonnemaker, J., DEMIS, 2002: a Dynamic Event Model for Interactive Systems. ACM Virtual Reality Software Technology 2002, Hong Kong. 
10. Lewis, $\mathrm{M}$ and Jacobson, Games Engines in Scientific Research. Communications of ACM, Vol. 45, No. I, January 2002. pp 27-31.

11. Rickel, J., and Johnson, W.L., Animated Agents for Procedural Training in Virtual Reality: Perception, Cognition and Motor Control. Applied Artificial Intelligence 13:342-382, 1999.
12. Skorstad, G. and K. Forbus. Qualitative and Quantitative reasoning about thermodynamics, Proceedings of the Cognitive Science Society. (1989).

13. Weld, D.S. and De Kleer, J. Readings in Qualitative Reasoning about Physical Systems, Morgan Kaufmann, 1990. 